\section{Disrupting the Electrical Circuit: New Onset Atrial Fibrillation in a Patient With Severe Acute Respiratory Syndrome Coronavirus 2 (SARS-CoV-}

\section{2)}

Sohab Radwan ${ }^{1}$, Owen Schwartz ${ }^{2}$

1. Internal Medicine, MedStar Washington Hospital Center, Washington, DC, USA 2. Internal Medicine/Hospital Medicine, MedStar Washington Hospital Center, Washington, DC, USA

Corresponding author: Sohab Radwan, sohabr7@gmail.com
Received 06/29/2020 Review began 07/02/2020 Review ended 07/04/2020 Published 07/09/2020

(c) Copyright 2020

Radwan et al. This is an open access article distributed under the terms of the Creative Commons Attribution License CC-BY 4.0., which permits unrestricted use, distribution, and reproduction in any medium, provided the original author and source are credited.

\begin{abstract}
In December 2019, an outbreak of pneumonia cases in Wuhan, China was attributed to a novel coronavirus that was eventually recognized as severe acute respiratory syndrome coronavirus 2 (SARS-CoV-2). Currently identified as coronavirus disease 2019 (COVID-19), it has been declared a pandemic by the World Health Organization given its rapid global transmission. Various cardiovascular complications have been reported, including heart failure, myocarditis, acute coronary syndrome and arrhythmias, both atrial and ventricular. Regarding arrhythmias, onset from time of infection is variable but usually ranges from several days to a week. We hereby present a case of a COVID-19 positive patient presenting with new onset atrial fibrillation.
\end{abstract}

Categories: Cardiology, Internal Medicine, Infectious Disease

Keywords: sars-cov-2, covid-19, arrhythmias, atrial fibrillation

\section{Introduction}

In December 2019, a cluster of cases of pneumonia of unknown cause were identified in Wuhan, Hubei, China that was eventually attributed to a novel enveloped coronavirus, currently named as severe acute respiratory syndrome coronavirus 2 (SARS-CoV-2) [1]. Now identified as coronavirus disease 2019 (COVID19), the World Health Organization has declared SARS-CoV-2 a public health emergency and pandemic given its rapid spread across several countries worldwide [2]. Several cardiovascular manifestations have been reported so far, including arrhythmias, heart failure, cardiogenic shock, fulminant myocarditis and acute coronary syndrome [3]. Several reports worldwide have demonstrated a high incidence of arrhythmias, specifically supraventricular, in the setting of widespread and systemic inflammation associated with COVID-19. The prevalence of arrhythmias and conduction system disease in patients with COVID-19 varies from population to population [4]. Several case reports have shown that onset of atrial fibrillation may vary from a few days up to a week from acquiring the infection; however, sometimes may be longer $[5,6]$. Regarding the mechanism, hypoxia and electrolyte abnormalities, both known to contribute to the development of acute arrhythmias, have been frequently reported in the acute phase of COVID-19 illness [4]. We hereby present a case of a COVID-19 positive patient presenting with new onset atrial fibrillation and shed light on the current literature and the proposed mechanisms and etiology.

\section{Case Presentation}

A 37-year-old male patient with no significant past medical history presented to the emergency department with left foot pain of one-day duration following direct trauma. No other symptoms were reported on presentation, including palpitations, chest pain, shortness of breath or dizziness. Of importance, the patient reports multiple family members testing positive for SARS-CoV-2 two weeks ago as well as himself having experienced mild flu-like symptoms several days ago. Regarding social history, the patient denied any current or prior tobacco smoking, alcohol consumption or other drug abuse. Initial vital signs were notable for heart rate of 90 beats per minute, blood pressure of $152 / 89 \mathrm{mmHg}$, respiratory rate of 20 breaths per minute and an oxygen saturation of $100 \%$ on room air. The patient's weight is 86 kilograms with a calculated body mass index of 26.8. A 12-lead electrocardiogram demonstrated an irregularly irregular rhythm consistent with atrial fibrillation (Figure 1). 


\section{Cureus}

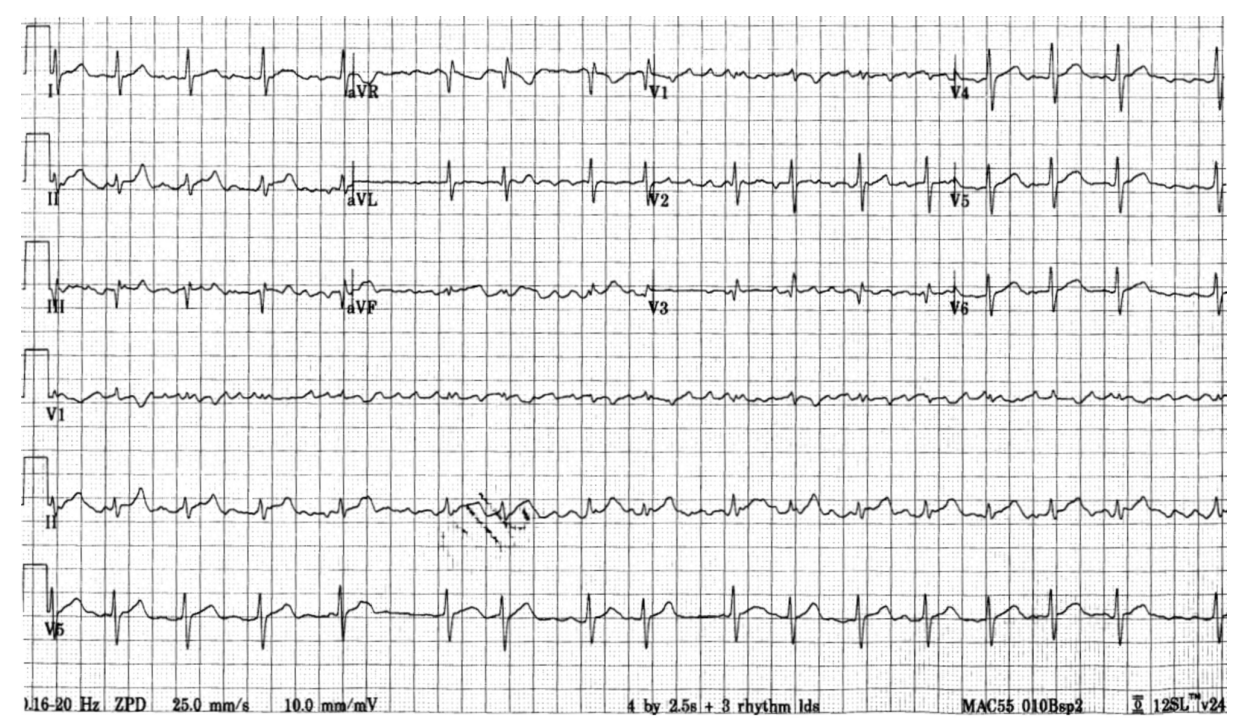

FIGURE 1: A 12-lead electrocardiogram demonstrating atrial fibrillation, calculated heart rate of 102 beats per minute, normal axis, no significant ST-segment deviation and non-specific T-wave abnormalities.

No baseline electrocardiogram was present in the medical chart. Pertinent diagnostic laboratory investigations demonstrated a normal level of troponin I, thyroid-stimulating hormone, hemoglobin and electrolytes, including potassium, magnesium and calcium (Table 1). 


\section{Cureus}

\begin{tabular}{|c|c|c|}
\hline Test & Value & Reference Range \\
\hline \multicolumn{3}{|l|}{ Cardiac testing } \\
\hline Troponin I (ng/mL) & $<0.017$ & $0.000-0.045$ \\
\hline \multicolumn{3}{|l|}{ General chemistry } \\
\hline Sodium (mmol/L) & 139 & 137-145 \\
\hline Potassium (mmol/L) & 4.1 & $3.5-5.1$ \\
\hline Chloride (mmol/L) & 105 & 98-107 \\
\hline Sodium bicarbonate (mmol/L) & 21 & $21-32$ \\
\hline Blood urea nitrogen $(\mathrm{mg} / \mathrm{dL})$ & 11 & $9-20$ \\
\hline Creatinine (mg/dL) & 0.77 & $0.66-1.50$ \\
\hline Glucose (mg/dL) & 131 & $65-140$ \\
\hline Calcium (mg/dL) & 9.0 & $8.5-10.1$ \\
\hline Magnesium (mg/dL) & 2.1 & $1.6-2.3$ \\
\hline \multicolumn{3}{|l|}{ Hematology } \\
\hline White blood cell count $(\mathrm{k} / \mu \mathrm{L})$ & 8.4 & $4.0-10.8$ \\
\hline Hemoglobin (g/dL) & 14.7 & $12.5-16.5$ \\
\hline Hematocrit (\%) & 43.5 & 37.5-49.5 \\
\hline 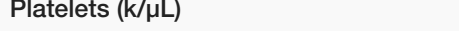 & 303 & $145-400$ \\
\hline \multicolumn{3}{|l|}{ Endocrinology } \\
\hline Thyroid-stimulating hormone (ulU/mL) & 1.150 & $0.400-4.000$ \\
\hline \multicolumn{3}{|l|}{ Infectlous disease } \\
\hline SARS-CoV-2 RNA PCR & Positive & Negative \\
\hline
\end{tabular}

\section{TABLE 1: Initial laboratory values}

SARS-CoV-2: severe acute respiratory syndrome coronavirus 2; RNA: ribonucleic acid; PCR: polymerase chain reaction

Initial evaluation by the podiatric surgery team revealed a left fifth proximal phalanx displaced fracture. Decision was to take the patient to the operating theater for open reduction internal fixation of this fracture. Per our hospital's policy for surgical procedures, a SARS-CoV-2 polymerase chain reaction test was done and resulted as positive. He eventually underwent the procedure without any complications. Unfortunately, no echocardiogram was obtained to investigate the presence of structural heart disease. No rate control agents were required throughout his hospitalization. CHA2DS2-VASc (congestive heart failure, hypertension, age $\geqslant 75$ years [double score], diabetes, prior stroke or transient ischemic attack [TIA; double score], vascular disease, age 65-74 years, sex class [female]) score for atrial fibrillation was zero, and therefore anticoagulation was not indicated. The patient was eventually discharged home with a new diagnosis of atrial fibrillation. Despite it being difficult to establish a causation between COVID-19 and atrial fibrillation in our patient, especially in the absence of an echocardiogram, this report aims to highlight the possible association between those two conditions.

\section{Discussion}

Cardiac arrhythmias have been reported in several recent reports in the literature in relation to hospitalized patients with COVID-19. Additionally, it was demonstrated to be associated with a higher risk of mortality [7]. However, the exact arrhythmic risk related to COVID-19 in patients with less severe illness or those who recover from the acute phase of the severe illness is currently unknown [4]. In regards to incidence, a report on 138 hospitalized COVID-19 patients in China has shown that $16.7 \%$ of patients developed cardiac arrhythmias [8]. Unfortunately, the exact nature of those cardiac arrhythmias was not reported in this study. However, given that sepsis in general has been associated with an increased rate of atrial arrhythmias, one would expect that those associated with COVID-19 to be mostly atrial as well [9]. Different types of 
arrhythmias have been reported so far including atrial fibrillation, atrial flutter, ventricular tachycardia as well as ventricular fibrillation. Interestingly, one report showed that arrhythmias were observed in $7 \%$ of patients who did not require intensive care unit treatment and in $44 \%$ of those who did [10].

Regarding pathophysiology, multiple mechanisms exist in which COVID-19 can trigger cardiac arrhythmias. Potential contributing factors include metabolic derangements, acidosis and hypoxia [11]. Moreover, the associated neurohormonal and catecholaminergic stress is thought to play a major role [11]. Similar to sepsis, the inflammatory cytokines and autonomic dysfunction associated with COVID-19 is thought to be a significant trigger for arrhythmias, especially atrial ones. Specifically, atrial fibrillation as a sequela of critical illness has been reported to occur in almost $10 \%$ of intensive care unit patients [12]. In addition, supraventricular tachyarrhythmias such as atrial fibrillation are associated more frequently with sympathetic nervous system activity in comparison to ventricular ones. Consequently, this explains the high prevalence of supraventricular tachyarrhythmias in patients with COVID-19 given the associated hyperbolic sympathetic activity [13].

It is noteworthy to mention that malignant cardiac arrhythmias, such as sustained ventricular tachycardia and ventricular fibrillation, have been observed in hospitalized patients with COVID-19. Despite the limited clinical and published data, this most likely occurs in the setting of COVID-19-associated acute cardiac injury and myocarditis [14]. Moreover, patients with inherited arrhythmia syndromes, such as long QT syndrome, may be at an increased proarrhythmic risk in the setting of COVID-19 infection, necessitating specialized care [15]. Improved understanding of this issue is critical, and further research is needed in guiding the need for additional arrhythmia monitoring post discharge and whether cardioverter defibrillators will be required in those with impaired left ventricular function attributed to COVID-19 [4].

\section{Conclusions}

In light of the emerging pandemic of COVID-19, several cardiovascular complications including arrhythmias are gaining worldwide recognition. Awareness of such entities and their theoretical pathophysiology is required for early recognition. Future research is needed to further investigate those observations and the associated clinical outcomes.

\section{Additional Information \\ Disclosures}

Human subjects: Consent was obtained by all participants in this study. Conflicts of interest: In compliance with the ICMJE uniform disclosure form, all authors declare the following: Payment/services info: All authors have declared that no financial support was received from any organization for the submitted work. Financial relationships: All authors have declared that they have no financial relationships at present or within the previous three years with any organizations that might have an interest in the submitted work. Other relationships: All authors have declared that there are no other relationships or activities that could appear to have influenced the submitted work.

\section{References}

1. Guan WJ, Ni ZY, Hu Y, et al.: Clinical characteristics of coronavirus disease 2019 in China . N Engl J Med. 2020, 382:1708-1720. 10.1056/NEJMoa2002032

2. World Health Organization. Coronavirus disease (COVID-19) outbreak. (2020). Accessed: July 8, 2020: https://www.who.int.

3. Sellers SA, Hagan RS, Hayden FG, Fischer WA II: The hidden burden of influenza: a review of the extrapulmonary complications of influenza infection. Influenza Other Respir Viruses. 2017, 11:372-393. 10.1111/irv.12470

4. Lakkireddy DR, Chung MK, Gopinathannair R, et al.: Guidance for cardiac electrophysiology during the coronavirus (COVID-19) pandemic from the Heart Rhythm Society COVID-19 Task Force; Electrophysiology Section of the American College of Cardiology; and the Electrocardiography and Arrhythmias Committee of the Council on Clinical Cardiology, American Heart Association [Epub ahead of print]. Heart Rhythm. 2020, e1-e9. 10.1016/j.hrthm.2020.03.028

5. Taha ME, Alsafi W, Taha M, Eljack A, Ibrahim H: Coronavirus disease and new-onset atrial fibrillation: two cases. Cureus. 2020, 12:e8066. 10.7759/cureus.8066

6. Kochav SM, Coromilas E, Nalbandian A, et al.: Cardiac arrhythmias in COVID-19 infection . Circ Arrhythm Electrophysiol. 2020, 13:e008719. 10.1161/CIRCEP.120.008719

7. Shi S, Qin M, Shen B, et al.: Association of cardiac injury with mortality in hospitalized patients with COVID-19 in Wuhan, China [Epub ahead of print]. JAMA Cardiol. 2020, 10.1001/jamacardio.2020.0950

8. Wang D, Hu B, Hu C, et al.: Clinical characteristics of 138 hospitalized patients with 2019 novel coronavirus-infected pneumonia in Wuhan, China. JAMA. 2020, 323:1061-1069. 10.1001/jama.2020.1585

9. Cheng P, Zhu H, Witteles RM, Wu JC, Quertermous T, Wu SM, Rhee JW: Cardiovascular risks in patients with COVID- 19: potential mechanisms and areas of uncertainty. Curr Cardiol Rep. 2020, 22:34. 10.1007/s11886020-01293-2

10. Liu K, Fang YY, Deng Y, et al.: Clinical characteristics of novel coronavirus cases in tertiary hospitals in Hubei Province. Chin Med J. 2020, 133:1025-1031. 10.1097/CM9.0000000000000744

11. Kuipers S, Klouwenberg PMK, Cremer OL: Incidence, risk factors and outcomes of new-onset atrial 


\section{Cureus}

fibrillation in patients with sepsis: a systematic review. Crit Care. 2014, 18:688. 10.1186/s13054-014-0688-5

12. Seguin P, Launey Y: Atrial fibrillation is not just an artefact in the ICU . Crit Care. 2010, 14:182.

10.1186/cc9093

13. Otake H, Suzuki H, Honda T, Maruyama Y: Influences of autonomic nervous system on atrial

arrhythmogenic substrates and the incidence of atrial fibrillation in diabetic heart. Int Heart J. 2009, 50:627641. 10.1536/ihj.50.627

14. Beri A, Kotak K: Cardiac injury, arrhythmia and sudden death in a COVID-19 patient [Epub ahead of print] HeartRhythm Case Rep. 2020, 10.1016/j.hrcr.2020.05.001

15. Wu CI, Postema PG, Arbelo E, et al.: SARS-CoV-2, COVID-19, and inherited arrhythmia syndromes [Epub ahead of print]. Heart Rhythm. 2020, 10.1016/j.hrthm.2020.03.024 